MS43-P13 Changes of the molecular structure in organic thin film transistors during operation

Fabiola Liscio ${ }^{1}$, Laura Ferlauto ${ }^{1}$, Micaela Matta ${ }^{2}$, Rafael Pfattner ${ }^{3}$, Mauro Murgia ${ }^{4}$, Conceptio Rovira ${ }^{3}$, Marta Mas-Torrent ${ }^{3}$, Francesco Zerbetto $^{2}$, Silvia Milita ${ }^{1}$, Fabio Biscarini ${ }^{5}$

1. CNR - IMM Sezione di Bologna, Via P. Gobetti 101, 40129 Bologna, Italy

2. Dip. di Chimica "G. Ciamician”, Università di Bologna, 40126 Bologna, Italy

3. Institut de Ciencia de Materials de Barcelona (ICMAB-CSIC) and Networking Research Center on Bioengineering, Biomaterials and Nanomedicine (CIBER-BBN), 08193 Bellaterra, Spain

4. CNR - ISMN Sezione di Bologna, Via P. Gobetti 101, 40129 Bologna, Italy

5. Dip. Scienze della Vita, Università di Modena e Reggio Emilia, 41125 Modena, Italy

email: liscio@bo.imm.cnr.it

Thin film of organic semiconductors have been widely studied at different length scales, for improving the electrical response of devices based on them. Up to now, a lot of knowledge has been gained about how molecular packing, morphology, grain boundaries and defects affect the charge transport in Organic Field Effect Transistors (OFETs) [1-3]. However, in real application, the impact of an electric field on the organic semiconductor and thus the transport parameters needs to be taken into account in order to develop high-performance organic device. Here, we present for the first time the structural evolution of pentacene thin film observed during the OFET operation. This investigation was achieved by performing X-Ray Diffraction measurements, both in out-of-plane and grazing incidence geometries, in real time, i.e. during the application of drain-source $\left(\mathrm{V}_{\mathrm{DS}}\right)$ and gate $\left(\mathrm{V}_{\mathrm{G}}\right)$ voltages. In particular, Selected Bragg reflections characteristic of the lattice planes perpendicular and parallel to the dielectric surface were monitored during OFET operation. The effect of $\mathrm{V}_{\mathrm{GS}}$ on the pentacene crystal structure was calculated by Molecular Dynamics and Density Functional Theory simulations. The interplay between the calculated changes of the structure and the experimental results provide an understanding of the structural evolution of operating transistors. The transversal field induces a slight change of orientation of the molecules within the crystalline domains, whereas it may be more relevant for a small fraction of molecules located at the grain boundaries or nearby whose re-orientation is substantial and leads to a significant energetic lowering of the charges. This implies that the re-oriented molecules at grain boundaries might act as shallow traps for charge carriers, opening a new unexplored route for understanding the relationship between structure and electrical properties of OFET devices.

[1] A. Shehu, S. D. Quiroga, P. D’Angelo,et al., Phys. Rev. Lett. 2010, 104, 246602

[2] J. Rivnay, L. H. Jimison, J. E. Northrup et al. Nat. Materials 2009, 8, 952

[3] F. Liscio, C. Albonetti, K. Broche t al., ACSNano, 2013, 7,1257

Keywords: real time X-ray diffraction, lattice strain, organic field-effect transistors, bias stress, pentacene
MS44. New applications of old algorithms in crystallography

Chairs: George Sheldrick, Isabel Uson

\section{MS44-P1 Macromolecular diffraction data: \\ Maximum entropy Patterson calculation used to estimate unobserved data}

Anders Kadziola ${ }^{1}$

1. Department of Chemistry, University of Copenhagen

email:kadziola@chem.ku.dk

The principle of maximum entropy has been applied to the Patterson function. Patterson functions are used in classical molecular replacement rotation functions as Patterson overlap functions. Larger portions of reciprocal space with unobserved data are likely to introduce serious errors in such overlap function calculations. Inclusion of maximum entropy estimates for missing intensities are shown to reduce these errors considerably. As a prior in the maximum entropy calculation, a Fourier transform of a parameterized fit to the average intensity as a function of resolution is used. The calculations are fully CCP4-compatible, reading and writing the $\mathrm{mtz}^{-}$and map-file format.

Keywords: maximum entropy, Patterson, molecular replacement 